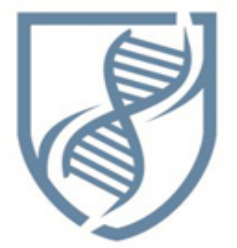

Journal of Bioscience and Applied Research

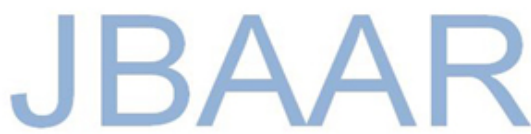

WWW.JBAAR.ORG

\title{
Light and electron microscopic observations on the development and structure of the inner ear in the brown-spotted grouper Epinephelus chlorostigma (Serranidae, Teleostei)
}

\author{
Mostafa A. Salem \\ Zoology Department, Faculty of Science, Zagazig University, Egypt \\ (Corresponding author e.mail: salemlab3@hotmail.com
}

\begin{abstract}
The development and structure of the inner ear were studied in the brown-spotted grouper Epinephelus chlorostigma, using light and electron microscopy. Light microscopy indicated: 1 ) The auditory or otic placode first appeared at 18 hours after fertilization (hatching time). It excavates forming a small sac called otic vesicle at 20 hours after fertilization. 2) At 24 hours (one day) after fertilization, the vesicle enlarged in size having a wide cavity with thin walls. 3) At two days after fertilization, the anterior and posterior semicircular canals seemed to be the first formed , while, the horizontal canal, the utricular and saccular cavities are differentiated at four days after fertilization. 4) At six days after fertilization, the saccular macula covered by an elongated otolith and the neuromasts of the ampulla in the semicircular canals are well established. 5) The crista ampullaris in the semicircular canals and the sensory regions in the utricular and saccular maculae become well visible and differentiated at nine days after fertilization. It was suggested that the hearing mechanism in the E. chlorostigma may be established early compared with other fishes. Electron microscopy on the sensory areas of the inner ear revealed: 1) The saccular macula contained two types of hair ciliary bundles; type C1 bundles characterized by short graded stereovilli and kinocilium that was approximately three times longer than the largest stereovillus and type C2 bundles consisted of a series of long graded stereovilli and a kinocilium that was no more than two times longer than the longest stereovillus. 2) The utricular macula contained type C3 bundles and type C4 ones. The type C3 bundles had a limited number of graded stereovilli and a kinocilium that had the same length of the longest stereovilli or somewhat longer, while the type C4 hair bundles consisted of numerous stereovilli with the same length and without a kinocilium. Three types of connectors (links) between the stereovilli were observed.
\end{abstract}

The different types of ciliary bundles and sites of connectors are possibly of functional importance.

Keywords: inner ear, sacculus, utriculus, hair cells, kinocilium, stereovilli

\section{Introduction}

Fish represent the largest group of vertebrates and display the greatest diversity of auditory structures. However, studies addressing how the form and function of the auditory system change during development to enhance perception of the acoustic environment are rather sparse compared to other vertebrate groups.

It was known that the acoustico-lateralis system of fish is composed of two major structures; the inner ear and the lateral line. The inner ear is responsible for the balance and detection of acoustic signals, whereas the lateral line detects water-borne vibration signals (Hawkins, 1986). These mechanosensory functions are crucial for the survival of fish. In terms of anatomical structure, the functional units of the inner ear are sensory hair cells and are used to detect underwater acoustical signals (Ramcharitar et al., 2004).

The sensory cells of the inner ear in fishes continue proliferating post-embryonically (Corwin, 1981). This characteristic potentially makes the inner ear of these vertebrates a unique model for studying the mechanisms involved in the differentiation of sensory cells. The structure of the adult fish inner ear (labyrinth) is well known, and it has been described for many different species of teleosts (Platt, 1983; Bang et al., 2001; Song et al., 2006). However, there are few studies on the development of the sensory organs in the inner ear (Sokolowski and Popper, 1987; Beccera and Anadon, 1993; Salem and Omura, 1998). The fish inner ear is divided into two regions, pars superior and pars inferior. The former responds primarily to movements of the body and postural 
changes, while the latter responds to both gravitate and acoustic stimuli (Popper, 2002). The pars superior comprises three semicircular canals and one fluid-filled pouch, the utriculus, while the pars inferior comprises one or two fluid-filled pouches, the saccule and lagena.

In the utriculus, sacculus and lagena, there are large areas of sensory epithelia which are termed the utricular, saccular and lagenar maculae, respectively. The saccular macula appears to be the major receptor of sound in most fishes (Lanford et al., 2000). Previous studies have shown that the sensory epithelium in each macula consists of sensory hair cells, supporting cells, transitional and/or precursor cells (Kawamura et al., 1989; Salem and Omura, 1998; Hertwig and Schneider, 1999; Ladich and Popper, 2001). Each hair cell possesses a hair bundle that is composed of two or more rows of stereovilli or stereovilli and a single true cilium, the kinocilium (Satir, 1977).

The stereovilli are interconnected by several types of connectors. They appear to stabilize the hair bundle mechanically, and may be directly involved in mechanoelectric transduction (Hachney and Furness, 1995). The sensory hair cell responds maximally when its hair bundle is deflected towards or away from its kinocilium (Little and Neugebauer, 1985). A deflection of the stereovilli towards the kinocilium leads to depolarization (excitation) of the hair cell, and deflection in the opposite direction leads to hyperpolarization (inhibition)(Hudspeth, 1983). Consequently, it is possible to determine the physiological polarization of a sensory epithelium by observations of the stereovilli (hair bundle) with the scanning electron microscope (Flock et al., 1977).

It is a common observation that hair bundles differ in the length and size in different regions of the sensory epithelium. Also, there are considerable variations in the number and lengths of the stereovilli borne by sensory cells in the inner ear, and in the length of the kinocilium. It has been proposed that this might have a functional significance (Platt and Popper, 1984).

Cross-links (connectors) appropriate for transmitting stimulatory force further from the kinocilium to the adjacent stereovilli have been described (Ernstson and Smith, 1986). The stereovilli of each hair bundle in the macular organs have also been shown to be interconnected (in lizard: Bagger-Sjoback and Wersall, 1973; in rat: Ross, 1974; in guinea pig: Pickles et al., 1984; in frog: Schmidt and Thurm, 1984; in human: Rhys Evans et al., 1985; in birds: Pickles et al., 1989; in fishes: Neugebauer, 1986; Salem, 2003). Therefore, the search for structural elements responsible for this sensory process has also concentrated on this area.

Epinephelus chlorostigma known as the brown-spotted grouper (family: Serranidae) and has been successfully cultured in a hypersaline water at Jeddah farming center, Saudi Arabia. This species as well as 22 other species under family Serranidae have higher market potential in several countries, especially in South-east Asia and in the Middle East, and they are commercially important with highly priced marine fish species (Heemstra and Randall, 1993).

In previous works, (Salem and Omura, 1998; Salem and Zaghloul, 2001; Salem and Al-Jahdali, 2006), we studied the development and ultrastructure of the inner ear sensory epithelia in relation to otolith and otolithic membrane formation in different fish species. We have observed that the saccular sensory epithelium was the first epithelium appearing in the inner ear in the most studied species referring to the importance of this part in the auditory sense. We also noted that different cells in the sensory epithelium produce various secretory materials which contribute in the formation of the otolith and/or otolithic membrane. Completion for the series of these studies, the present research is aimed to obtain information on the early development of the inner ear, collecting data on the morphological arrangement of the sensory hair bundles and to determine the various links between the stereovilli in the saccular and utricular sensory epithelia of the bottom feeder fish Epinephelus chlorostigma, using light and electron microscopy (scanning and transmission). This study is of considerable interest because it is the first report to describe the embryonic development of the inner ear in this commercially valuable species.

\section{Materials and Methods}

The specimens of brown-spotted grouper, Epinephelus chlorostigma were obtained from the fish broodstock of Jeddah Faming Center located on the Red Sea coast of Jeddah, Saudi Arabia. In addition to adult fish samples, seven different embryonic stages; 18 hours (hatching time, at water temperature $23-25{ }^{\circ} \mathrm{C}$ ), 20 hours and 1, 2, 4, 6, 9 days after fertilization, were collected. The whole samples of the embryos were fixed in Bouin's solution, dehydrated in increasing ethanol series and embedded in paraffin wax from 2-4 hours, depending on the size of the specimens. The chosen samples were serially sectioned at 3-5 microns. The sections were stained with Mallory's triple stain and Ehrlich's haematoxylin counterstained by eosin and examined under the light microscopy. The steps were proceeded according to the method of Bancroft and Gamble (2002).

Larval specimens at nine days after fertilization were also selected and fixed in $1 \%$ glutaraldehyde in $0.1 \mathrm{M}$ phosphate buffer for $2 \mathrm{~h}$. Following several rinses in phosphate buffer containing 7\% sucrose, the specimens were pos-fixed in $1 \%$ osmium tetroxide in $0.1 \mathrm{M}$ phosphate buffer. After dehydration through a graded series of ethanol, the specimens were infiltrated with araldite resin. For transmission electron microscopy observations, ultrathin sections $(80-90 \mathrm{~nm})$ at the inner ear region were obtained using sharp glass knives. The sections were mounted on electron-microscope grids, stained with uranyl acetate and lead citrate and examined on a JEOL transmission electron microscopy.

For scanning electron microscopy, adult specimens of E. chlorostigma were captured and anesthetized. The crania were opened dorsally, the brain removed, and 
through a small opening in the inner ear, a small amount of 2\% glutaraldehyde in phosphate buffer was placed for 30 minutes. The inner ear was dissected, and the otoliths and the otolithic membrane were removed. The sacculus is gently cut, rinsed with $0.1 \mathrm{M}$ phosphate buffer containing a dilute solution of $\mathrm{MgCl}_{2}$, and fixed in 2\% glutaraldehyde + $2 \%$ tannic acid in the same buffer on ice. After 15-30 min on ice the tissue was washed with the buffer, postfixed in $1 \%$ osmium tetroxide for 15 minutes, washed, and incubated for $20 \mathrm{~min}$ in $2 \%$ tannic acid. The tissues were then dehydrated through ascending ethanol series, dried by the critical point drying technique, using carbon dioxide, mounted on stubs and coated with gold in a JEOL fine coat Ionsputter, and examined with JEOL JSM-35 scanning electron microscope. The technique of the electron microscopy was proceeded according to the method of Robenson et al. (1987).

\section{Results}

\section{1) 18 hours after fertilization (hatching time):}

The inner ear (membranous labyrinth) appears as a pair of ectodermal thickenings known as auditory or otic placode on either side of the hind brain, just as in other vertebrates. The otic placode can first be recognized as an oval grouping of cells within the ectoderm (Fig. 1).

\section{2) 20 hours after fertilization:}

The placode is rapidly converted into a hollow vesicle (otic vesicle). The vesicle has narrow cavity and thick walls composed of several layers of polygonal cells (Fig. 2). The process differs from that seen in higher vertebrates such as chick (Alvarez et al., 1989), in which the placode invaginates as an epithelial sheet to form a cup before pinching off from the surface ectoderm as a closed vesicle sunk within the head mesenchyme. In $E$. chlorostigma, by contrast, no cup or pit is seen and the initial oval grouping of cells in the ectoderm appears simply to thicken and model itself into an ovoid solid ball just beneath the surface. The cell nuclei move radially towards the surface of this ball, and the cells which contacted at the centre begin to be lost, so that a small slitlike space appears, referring to the first sign of the ear lumen (the otic vesicle).

\section{3) One days (24 hours) after fertilization:}

The otic vesicle enlarges by expanding its lumen, remaining a simple ovoid shape for about 24 hours, and its walls become thinner (Fig. 3). The first sign of segmentation is the appearance of an evagination at the rostral region of the otic vesicle (Fig. 3, arrows).

\section{4) Two days (48 hours) after fertilization:}

At this time, the otic vesicle embarks on a series of shape changes that convert it into a complex structure with three semicircular canals more or less perpendicular to each other (Figs 4\&5). The process begins with the development of protrusions that poke inwards from the walls of the vesicle into the lumen (Figs $4 \& 5$, arrows). The first protrusion appears from the posterior part of the lateral wall which bifurcates into two protrusions projecting into the otic lumen (Fig. 4, arrows), while a second one appears from the middle of the posterior wall (Fig. 5, arrows). Each protrusion consists of a thin layer of otic epithelium covering a finger-shaped core of acellular matrix; as the volume of matrix increases, the all protrusions gradually elongate. The spaces resulting from these protrusions become the semicircular canals; the anterior and posterior canals seemed to be the first formed (Fig. 5). The saccular and utricular cavities can be established. The saccular cavity evaginates medially forming a small pocket (Fig. 5).

\section{5) Four days after fertilization:}

In this stage the three semicircular canals and the utriculus become well separated (Figs 6\&7). The first indication of the future sensory maculae is the appearance of a thickened area in the median wall of the saccular pocket referring to the establishment of the saccular macula (Fig. 7, arrows). There is no sign for appearance of the utricular macula in this stage.

\section{6) Six days after fertilization:}

The cristae ampullaris of the semicircular canals appeared as a thickened epithelium like a bell-shaped structure, the ampulla of the horizontal canal is the first to be formed, but not yet differentiated (Fig. 8). The cristae seemed to be formed after complete separation of the three semicircular canals. At this stage also, the saccular otolith, overlaying the saccular macula, is first appeared as an elongated structure (Fig. 9). The inner ear has become partly encased in a cartilage capsule, with thick plates separating it from the pharynx ventrally and isolating its posterior part from the neural tube medially (Figs $8 \& 9$, arrows).

\section{7) Nine days after fertilization:}

This stage represents an advanced step in the development of the inner ear where all the major structures of the inner ear are clearly visible and differentiated (Fig. 10 ). The lagena is the last ear pockets to appear. It appears to be formed from a ventromedial caudal evagination of the saccular cavity (Fig. 11). The sensory epithelia in the ampullae of the semicircular canals and in the saccular and utricular maculae are well differentiated (Figs 12, 13\& 14).

Because this stage represented an advanced step in the saccular and utricular sensory epithelia differentiation, it was important to examine them under the transmission electron microscope. Two types of cells are clearly observed in the utricular and saccular sensory epithelia; hair (sensory) cells and supporting cells (Figs 15\&16). The hair cells are basically columnar but are slightly narrowed apically, possibly due to the expansion of the adjacent supporting cells at this level. Projecting from the luminal surface of each hair cell is a hair bundle which composed of numerous stereovilli and a single kinocilium (Figs $15 \& 16)$. The fine structure of the hair cells in the saccular and utricular maculae seems similar, except in the saccular macula each hair bundle appeared to be planted in a cuticular plate which is a homogenous structure occupying the apical region of the hair cell (Fig. 16). Cellular organelles are abundant in each sensory hair cell; mitochondria are the most abundant, especially in the supra-nuclear part of the cell (Figs 15\&16). 


\section{8)Morphology of the hair bundles in the adult forms:} The saccular hair bundles:

The saccular macula contains two types of hair ciliary bundles; type C1 bundles characterized by short graded stereovilli and a kinocilium that is approximately three times longer than the largest stereovilli (Fig. 17) and type C2 bundles consisted of a series of long graded stereovilli and kinocilium that is no more than two times longer than the longest stereovilli (Fig.18). The C1 hair bundles are abundant ate the peripheries of the macula while C2 bundles occurred at the centre.

The utricular hair bundles: The utricular macula contains type C3 hair bundles and type C4 ones. Type C3 hair bundle has a limited number of graded stereovilli and a kinocilium that has the same length of the longest stereovilli or somewhat longer (Fig. 19), while the type C4 hair bundle consisted of numerous stereovilli with the same length and without a kinocilium (Fig. 20). The C3 hair bundles are observed at the centre while the $\mathrm{C} 4$ bundles at the peripheries of the macula.

\section{9) The interconnections (links) in the hair bundles:}

Serial sections from the saccular macula revealed four types of connectors occurred between the components of the hair bundles: 1) oblique tip connectors which link the tips of the stereovilli (Figs 21\&22); 2) basal connectors of small filaments arising between the bases of the stereovilli (Fig. 23); 3) shaft connectors link the stereovilli with each other at the middle region which locates between the tip and basal connections (Fig. 24); and 4) horizontal connectors link the stereovilli with the kinocilium (Fig. 25). The kinocilium is characterized by presence of a number of microtubules.

\section{Discussion}

The ear development begins with the creation of an otic vesicle from a placodal thickening in the head ectoderm. In birds and mammals, the placode invaginates to become a cup and finally pinches off from the surface ectoderm as a hollow vesicle of internalized epithelium (Alvarez et al., 1989). In the fish (including the present study), however, the otic vesicle is formed in what appear to be a very different way: the placode condenses into a solid ball of cells, and a slit-shaped cavity then is formed at the centre of this ball; as the mass of cells becomes hollowed out and takes on an epithelial arrangement, it separates from the overlying epidermis.

In E. chlorostigma, the otic placode becomes visible at 18 hours after fertilization; an otic vesicle is formed by 20 hours; the vesicle losses its thickening and becomes thinner for future segmentation at one day after fertilization; the anterior and posterior canals as well as the utricular and saccular cavities are visible by two days; the saccular macula and the crista in the semicircular canals are established at four and six days, respectively. The differentiation of the sensory cells lining the macular organs and the semicircular canals is clearly seen at nine days after fertilization. These results indicated that the inner ear (membranous labyrinth) of the brown-spotted grouper Epinephelus chlorostigma is created quickly but mature slowly; i.e. an acoustic response and an ability to orientate relative to gravity can be occurring for the young larvae.

The semicircular canals develop in teleosts from invaginations in the otic vesicle wall (Becerra and Anadon, 1993; Haddon and Lewis, 1996; the present results). The order of development of the semicircular canals varies with species: three different patterns have been observed in osteichthyes. In the primitive bony fish Polypterus senegalus (Thomot and Bauchot, 1987), the anterior semicircular canal is the first to be formed and the posterior one the last. In zebrafish, Brachydanio rerio (Waterman and Bell, 1984) and Salmo trutta fario (Becerra and Anadon, 1993), the semicircular canals are formed in the same order as in E. chlorostigma (the present result): the first is the anterior and the last is the horizontal canal. This pattern appears to be the most frequent in teleosts. However, it is not common to all teleosts; the horizontal canal being the first to appear in Scophthalmus maximus (Becerra et al., 1990). This difference probably is species specific character.

It has been recorded that each semicircular canal has a spherical expansion known as the ampulla at one end. Within the ampulla there is a sensory epithelium known as the crista (Platt and Popper, 1981). In E . chlorostigma the crista ampullaris of the horizontal canal was the first to be formed. The same order is found in Salmo trutta fario (Becerra and Anadon, 1993) and Plecoglossus altivelis (Salem and Omura, 1998). The early formation of the horizontal crista may be related to the importance of this crista in the coordination of lateral body flexions in early larvae.

Some studies have been reported that the inner ear in fish begins with the formation of the saccular epithelium followed by formation of the utricular epithelium and the semicircular canals (Jenkins, 1977; Salem and Omura, 1998; Salem and Zaghloul, 2001). The development of the inner ear in E. chlorostigma doesn't follow this general sequence, where the anterior and posterior canals and the saccular and utricular cavities are appeared at the same time (all at four days). A similar result has been recorded in other fishes (Sokolowski and Popper, 1987; Becerra and Anadon, 1993). These developmental differences probably depend on the behavioral and habitual requirements of the fish. In addition the saccular macular epithelium in $E$. chlorostigma is established at four days after fertilization, before the utricular macula and the ampulla of the semicircular canals, suggesting that the auditory sense is probably established earlier than the sense of equilibrium. However, in other teleost species, such as Paralichthys olivaceus (Kawamura and Ishida 1985) and Epinephelus fuscoguttatus (Salem and Al-Jahdali 2006) the equilibrium sense is established earlier than the auditory sense, where the utricular macula appeared prior to the saccular one. These developmental differences may depend on the behavioral and habitual requirements of the fish.

The first obvious sign of development of sensory patches in the E. chlorostigma is the formation of the saccular otolith which become visible at 6 days after fertilization and enlarges gradually. From studies in other 


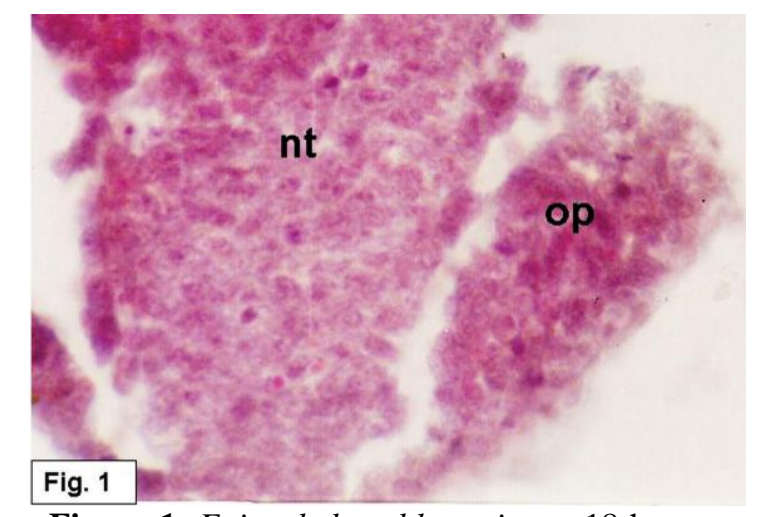

Figure 1. Epinephelus chlorostigma, 18 hours after fertilization. LM micrograph of a transverse section across the developing inner ear, showing the first appearance of the otic placode (op). nt, neural tube. X 450

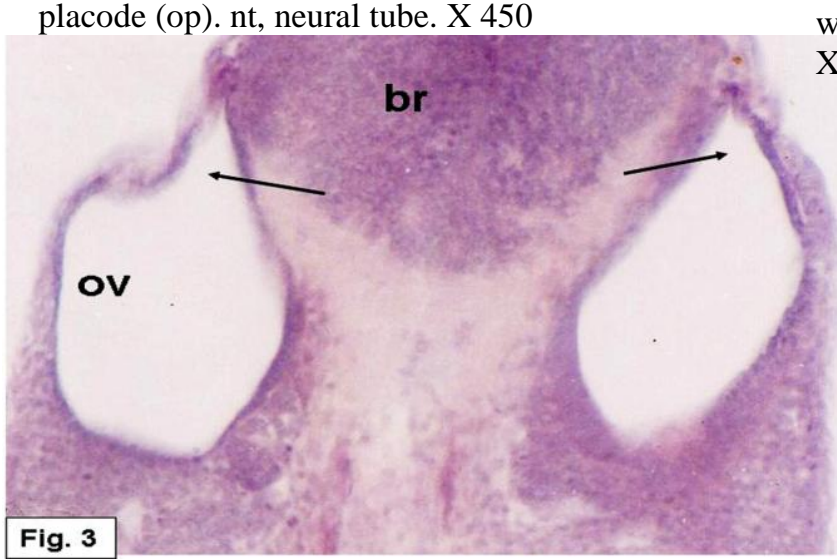

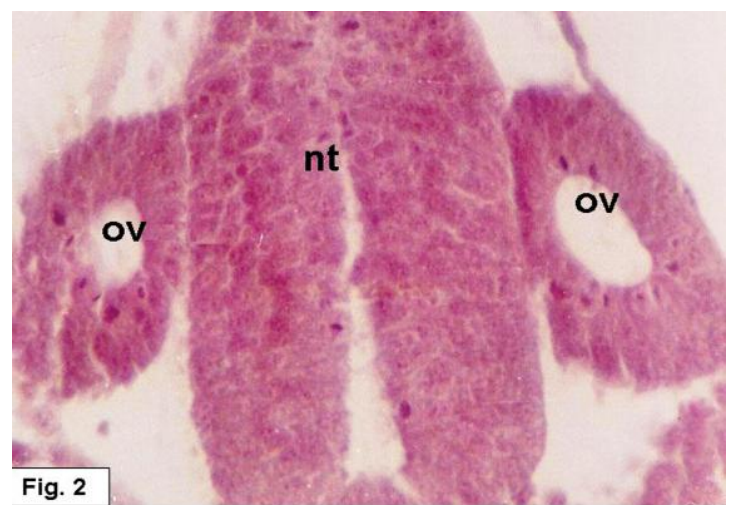

Figure 2. Epinephelus chlorostigma, 20 hours after fertilization. LM micrograph of a transverse section across the developing inner ear, showing the otic vesicle (ov) which has a narrow cavity and a thick wall. nt, neural tube. X 450

Figure 3. Epinephelus chlorostigma, one day (24 hours) after fertilization. LM micrograph of a transverse section across the developing inner ear, showing appearance of an evagination (arrows) at the rostral region of the otic vesicle (ov). br, brain. X 450

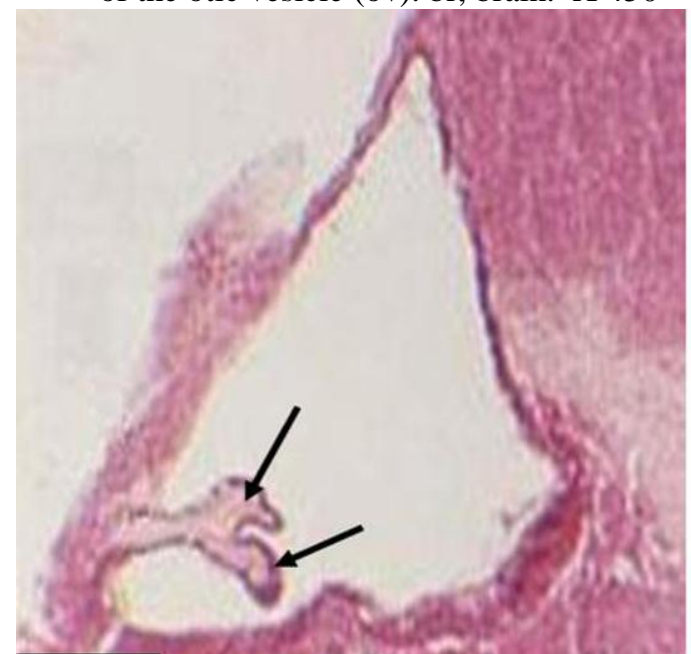

Fig. 4

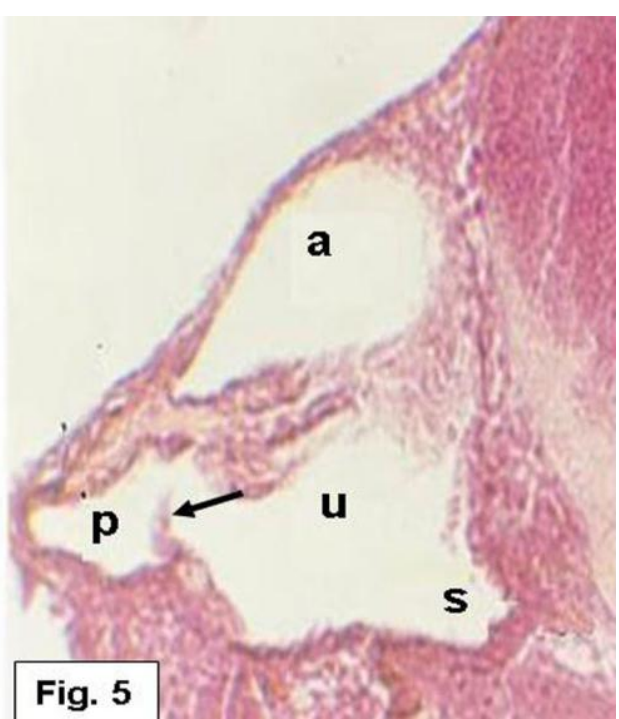

Figures 4\&5. Epinephelus chlorostigma, two days after fertilization. LM micrographs of transverse sections across the developing inner ear, showing establishment of the semicircular canals, the sacculus and utriculus. Fig. 4, showing a protrusion appears from the posterior part of the lateral wall which bifurcates into two protrusions (arrows) (X 150 ). Fig. 5, showing a protrusion (arrow) originates from the middle of the posterior wall of the otic vesicle. The saccular (u) and utricular (s) cavities can be established. a, anterior semicircular canal; p, posterior canal. X 220 

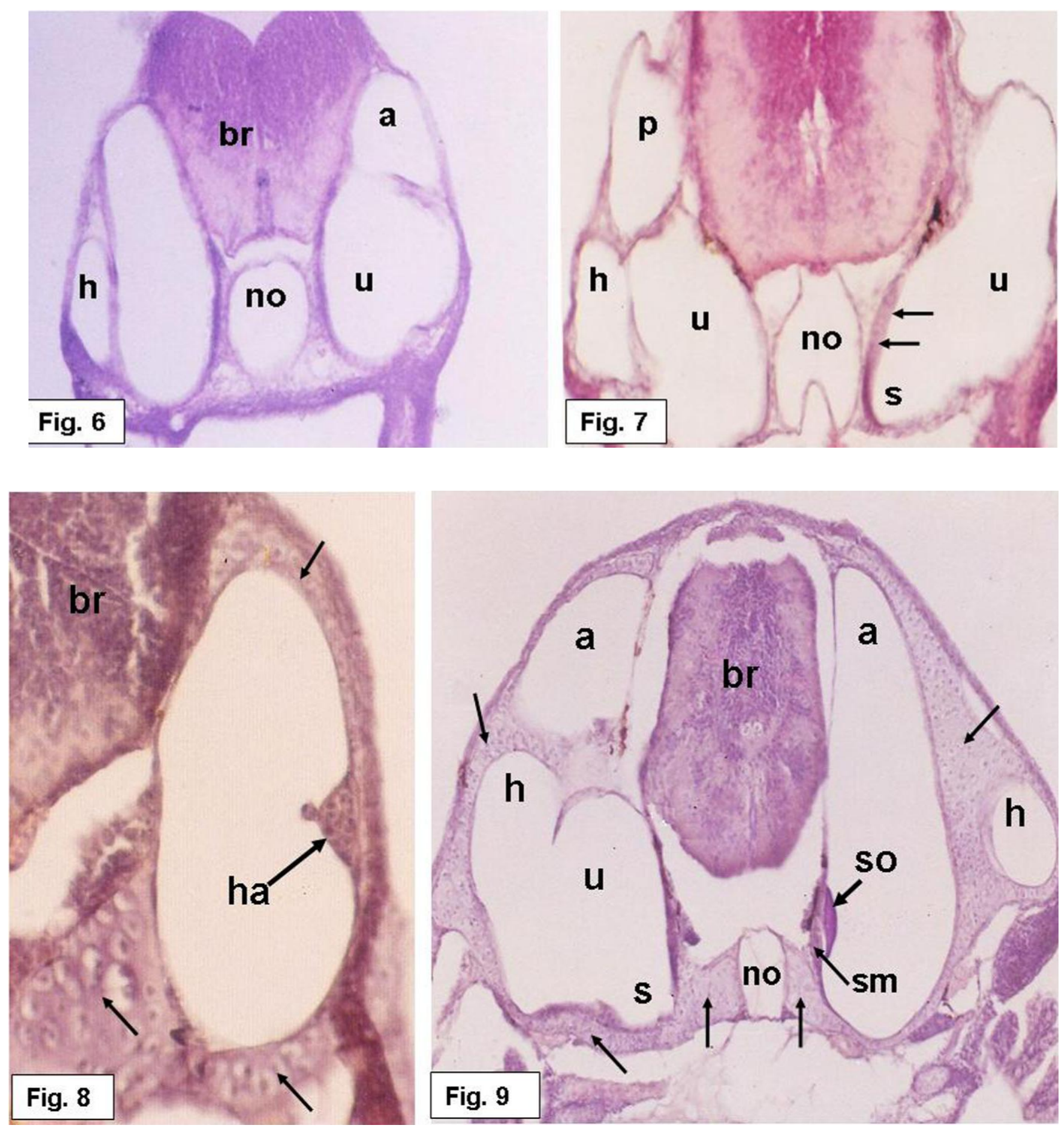

Figures 6\&7. Epinephelus chlorostigma, four days after fertilization. LM micrographs of transverse sections across the developing inner ear. The anterior (a), posterior (p) horizontal (h) semicircular canals and the utriculus (u) are completely established. The primordium of the saccular macula (arrows) can be seen in Fig. 7. br, brain; no, notochord; s, sacculus. Fig. 6, X 100; Fig. 7, X 140

Figures 8\&9. Epinephelus chlorostigma, six days after fertilization. LM micrographs of transverse sections across the developing inner ear, showing first appearance of the crista ampullaris in the horizontal canal (h) (Fig. 8) and the saccular otolith (so) overlying the saccular macula (sm) (Fig. 9). Note the ear components are surrounded by a cartilage capsule (arrows). a, anterior canal; br, brain; h, horizontal canal; no, notochord; s, saccule; u, utricle. Fig. 8, X 420; Fig. 9, X 150 

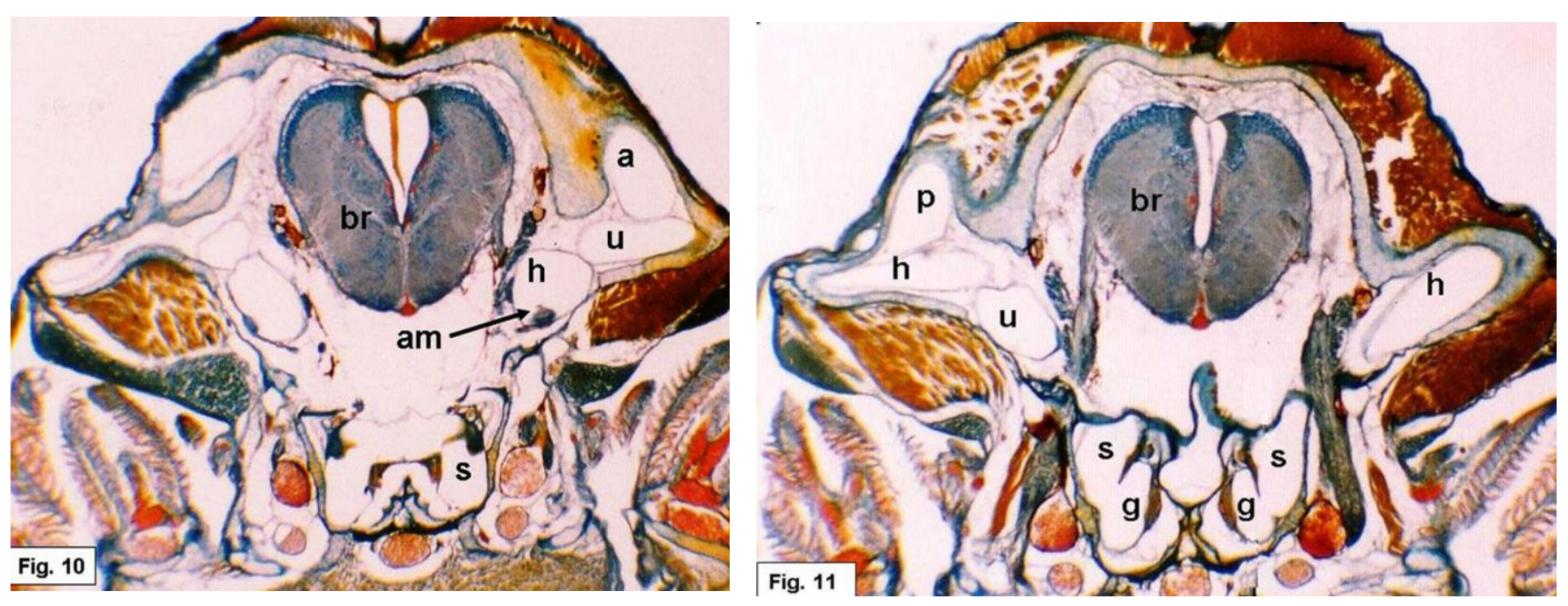

Figures 10\&11. Epinephelus chlorostigma, nine days after fertilization. LM micrographs of transverse sections across the developing inner ear. All the inner ear components seemed to be formed and separated. The lagena (g) appears to be formed from the ventromedial caudal evagination of the saccular cavity (s). The anterior (a), posterior (p) horizontal (h) semicircular canals and the utriculus (u) are clearly seen. am, ampulla of the horizontal canal; br, brain. X 50

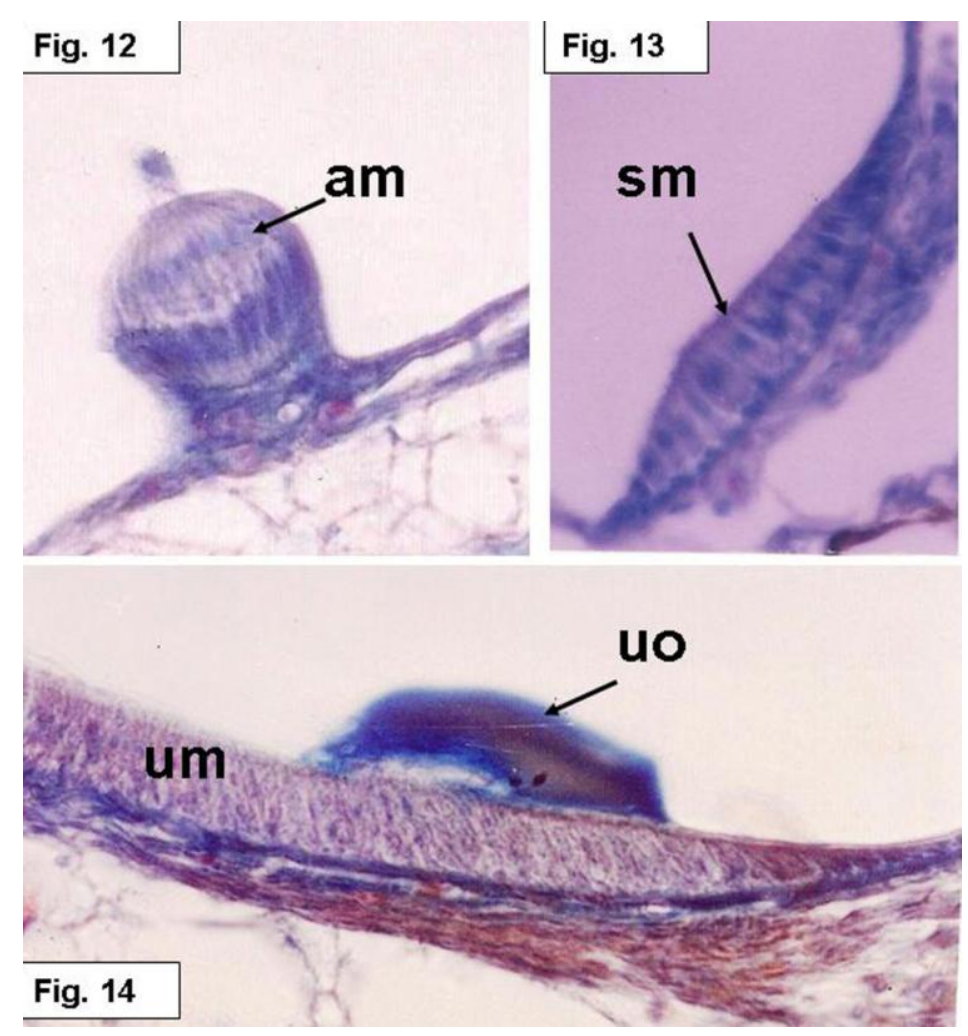

Figures 12,13\&14. Epinephelus chlorostigma, nine days after fertilization. LM micrographs of transverse sections across the developing inner ear, showing differentiation of the sensory region in: crista ampullaris (am) (Fig. 12), saccular macula (sm) (Fig. 13) and utricular macula (um) (Fig. 14). Note the utricular otolith (uo) overlain the utricular macula (um). Fig. 12, X 300; Fig. 13, X 450 ; Fig. 14, X 350. 

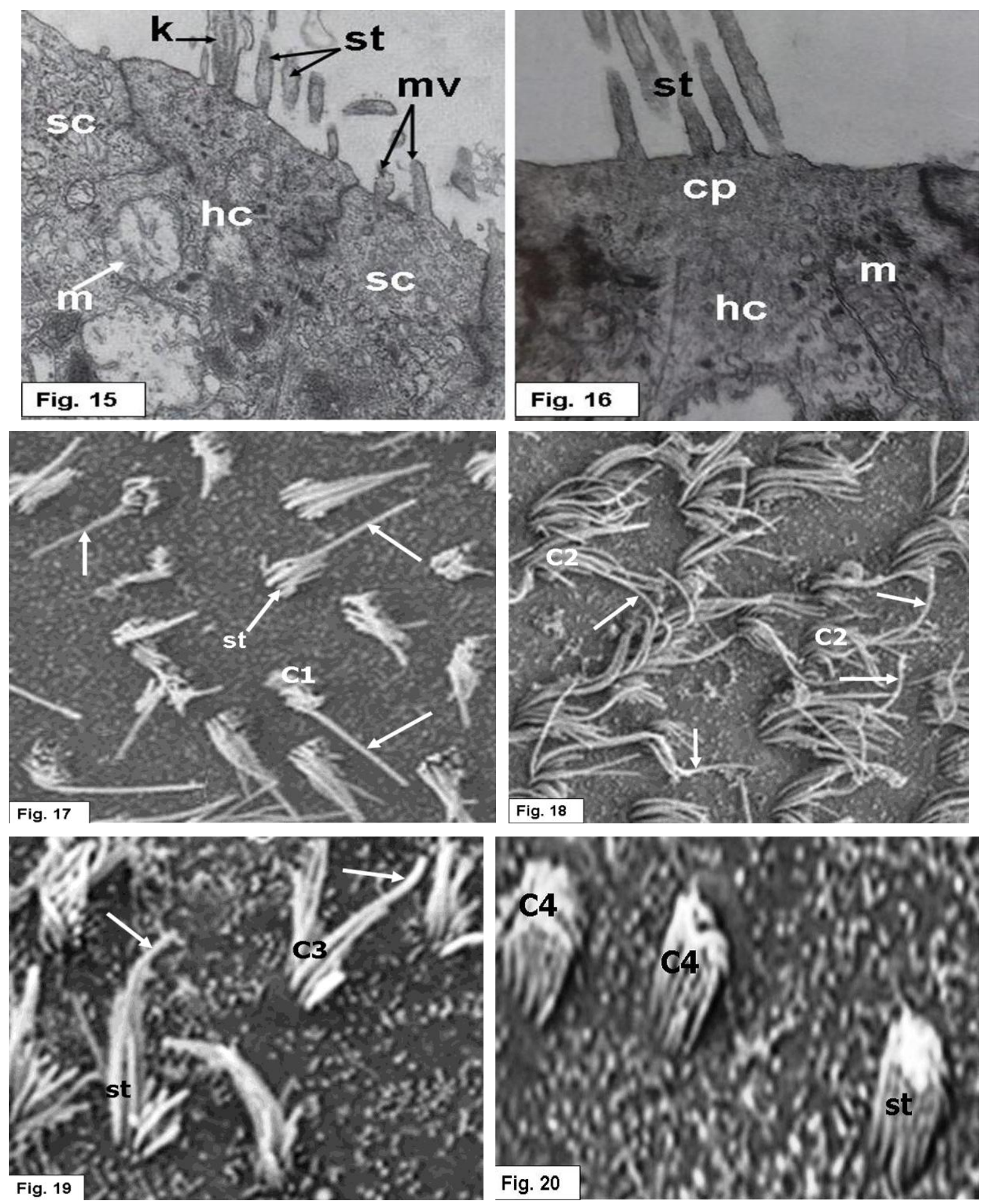

Figures 15\&16. Epinephelus chlorostigma, nine days after fertilization. TEM micrographs of the apical surface of the utricular and saccular maculae, respectively. Fig. 15, showing supporting cell (sc) and hair cell (hc) that provided with a number of stereovilli (st) and single kinocilium (k). Note some supporting cells (sc) bear microvilli (mv). m, mitochondria. X 9,500.Fig. 16, showing a hair cell (hc) bearing long stereovilli (st). The stereovilli are planted in a cuticular plate (cp). m, mitochondria. X 9,500

Figure 17. Epinephelus chlorostigma, adult specimen. SEM micrograph of the saccular sensory epithelium, showing $\mathrm{C} 1$ hair bundles at the peripheries of the epithelium, each one consisted of numerous short stereovilli (st) and a long kinocilium (arrows). X 4,100

Figure 18. Epinephelus chlorostigma, adult specimen. SEM micrograph of the saccular sensory epithelium, showing C2 hair bundles at the centre of the epithelium, each one consisted of numerous long stereovilli (st) and a long kinocilium (arrows) which is twice as long as the longest stereovilli. X 4,100

Figure 19. Epinephelus chlorostigma, adult specimen. SEM micrograph of the utricular sensory epithelium, showing C3 hair bundles at the centre of the epithelium. Each one consisted of a limited number of graded stereovilli (st) and single kinocilium (arrows) which is longer than the longest stereovilli. X 4,200

Figure 20. Epinephelus chlorostigma, adult specimen. SEM micrograph of the utricular sensory epithelium, showing C4 hair bundles at the periphery of the epithelium. Note the stereovilli (st) have the same length. X 4,200 

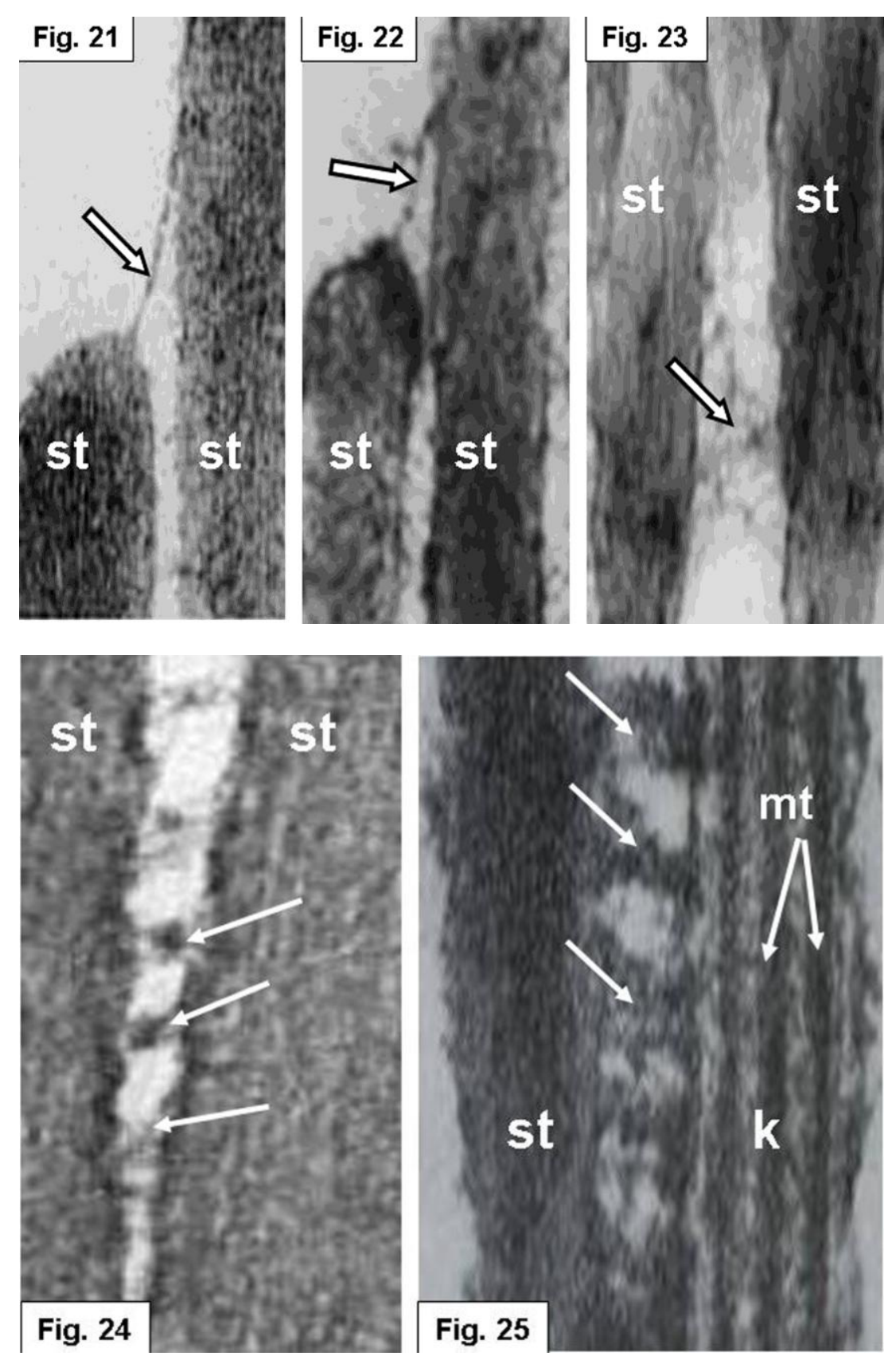

Figures 21-23. Epinephelus chlorostigma, adult specimen. TEM micrographs of sensory hair bundles from the saccular macula, showing different types of cross-links (connectors).

Figs 21\&22. Vertical or tip connectors (outlined arrows) between two stereovilli (st). X 33,000

Fig. 23. Basal connector (outlined arrow) between two stereovilli (st). X 33,000

Figures 24\&25. Epinephelus chlorostigma, adult specimen. TEM micrographs of sensory hair bundles from the saccular macula, showing different types of cross-links (connectors).

Fig. 24. Shaft connector (arrows) between two stereovilli (st). X 34,000

Fig. 25. Shaft connector (arrows) between a stereovillous (st) and a kinocilium (k). Note the microtubules (mt) in the kinocilium. X 33,500 
species (Campana and Neilson, 1985; Sokolowski, 1986; Saitoh and Yamada, 1989), it seems likely that the sited of otolith formation reflect local specialization of the epithelial cells: the cells of the sensory epithelium secrete an extracellular matrix (the otolithic membrane) within which calcium carbonate precipitates. By nine days after fertilization, and after formation of the saccular otolith, differentiated hair cells were clearly seen. The otolith has also observed, before differentiation of the hair cells, at 3 and 4 days after fertilization in the killifish (Radtke and Dean, 1982) and in the ayu (Salem and Omura, 1998), respectively. This suggests that the otolith formation probably necessary to initiate differentiation of the hair cells in the maculae.

The internal structure of the hair cells in the inner ear of E. chlorostigma is probably similar to the hair cells found in the vestibular organs of many higher vertebrates; however, the dimensions of the hair cells are almost double those found in the end organs of teleost fish (Lovell, 2006).

It has been reported that the stereovilli in the hair bundles are essential to the process of sensory transduction (Hudspeth, 1983). The present electron microscopic observations showed differences in lengths of the stereovilli and also in lengths of the kinocilia. In $E$. chlorostigma, four types of hair bundles are observed; C1 and C2 bundles in the saccular macula, and C3 and C4 in the utricular macula. The C1 bundles which have short stereovilli and long kinocilia have also been observed in the sciaenid fishes (Ramcharitar, et al. 2001), but C2 bundles are provided with long stereovilli and a kinocilium which no more than two times longer than the longest stereovilli. The C2 bundles of long stereovilli have also been observed in the inner ear end organs of sciaenid fishes (Ramcharitar, et al., 2001) and in some cartilaginous fish species, including Acipenser fulvescens (Lovell et al., 2005). However, C3 bundles having a limited number of graded stereovilli and a kinocilium, that has the same length of the longest stereovilli or somewhat longer, has been reported in the common goldfish Carassius auratus (Platt, 1977). Saunders and Dear (1983) mentioned that the bundles with long stereovilli and long kinocilium may be tuned to low frequencies, while bundles with shorter stereovilli may be tuned to higher frequencies. However, Platt and Popper (1984) proposed that the differences in the length of the ciliary bundles may be related to the nature of the stimulus applied to the ear fluid and tissues. However, the role of the C4 bundles which lack kinocilia may be to stabilize the other hair bundles as a whole.

Distribution of various hair cell bundle types in $E$. chlorostigma showed patterns similar to those reported for a number of other species (e.g., Popper, 1977, 1981; Song et al., 2006). C1 hair bundles are abundant on the periphery of the saccular macula. These bundles have kinocilia that are a few times longer than the longest stereovilli. It can postulate that the long kinocilia of such bundles interact with the edges of the otolith through the otolithic membrane.
There are some data to suggest that hair cell number affects auditory sensitivity (Corwin, 1983). However, other data suggest that hair cell densities are irrelevant to such sensitivity (Edds-Walton et al., 1999; Song et al., 2006). According to the present scanning electron microscopy observations, the saccular macula of E. chlorostigma seems to be possessed a high density of hair cells and hair bundles. If bundle density is an indicator of auditory sensitivity, then one might suppose that the E. chlorostigma shows the high specialization in hearing ability. Hudspeth and Jacobs (1979) and Schulz-Mirbach (2010) have shown that sensory transduction take place within the hair bundle on the hair cell. In the hair bundle the kinocilium primarily conveys mechanical displacements from the otolith to the stereocilia and drives the cell transduction process, leading to a depolarization in the transmembrane potential. Therefore, the transmission of force within the hair bundle is of particular importance. The hairs within a bundle are interconnected by some morphologically and topographically distinct links or connectors. These links fall into two categories: those linking the kinocilium to the stereovilli, and those interconnecting the stereovilli to each other (tip, shaft and basal connectors).

The tip or vertical connectors were presumed to affect membrane channels by concentrating force onto small membrane areas (Schmidt and Thurm, 1984). The location of the tip vertical connectors is compatible with electrophysiological results that favor the tip region as the site for transduction (Hudspeth, 1982). Tip connectors have been found in the cochlear and vestibular hair cells of the guinea-pig (Osborne et al., 1984; Pickles et al., 1984), the sacculus of frog (Schmidt and Thurm, 1984) and the sacculus, utriculus (Platt and Popper, 1984; Neugebauer and Thurm, 1984) and ampullae (Nagel et al., 1991) of fish. Osborne et al. (1984) and Pickles et al. (1984) have proposed that depolarization is associated with the stretching of these connectors when the otolithic membrane pulls the kinocilium away from the hair bundle, and that hyperpolarization is associated with their relaxation when the kinocilium is pulled toward the hair bundle. However, the basal and shaft connectors are not directly involved in mechano-electric transduction, but their role may be to stabilize the hair bundle as a whole (Hudspeth, 1982).

In conclusion: the present study revealed that the otic vesicle, the saccular macula and saccular otolith appeared at early stages, suggesting that the auditory sense in E. chlorostigma may be important for the larvae than the equilibrium sense which appears later. Presence of different types of hair bundles in the inner ear of E. chlorostigma might utilize bidirectionality for initiating highly specific excitatory and inhibitory reflexes to environmentally related stimuli. This could serve to enhance audition in this species. The hairs within a bundle are interconnected by some morphologically and topographically distinct connectors. This differentiated system of connectors exists that distributes force within the hair bundle; additional histochemical or immunohistochemical studies regarding their mechanical properties are required in order to explore 
their functional consequences. Several questions remain to be answered. Most important are questions dealing with why sensory cells develop selectively in specific areas of the early otocyst, and the mechanisms causing their induction. Furthermore, we do not know the causative factors directly inducing the cells to take up their adult hair cell orientation patterns. Further investigations on the development of structure and function of the fish auditory system are recommended in order to obtain a deeper understanding of how ontogenetic morphological changes in the auditory pathway relate to modifications in acoustic reception, auditory processing, and the capacity to communicate acoustically.

Acknowledgements. The author very grateful to Prof. Dr. Momtaz Hashim Ismail and Prof. Dr. Abd Albadei El-Attar for their careful reading of the manuscript and valuable discussions.

\section{References}

Alvarez, I.S., Martin- Partido, G., RodriguezGallardo, L., Gonzalez-Ramos, C. (1989). Cell proliferation during early development of the chick embryo otic anlage: quantitative comparison of migratory and non-migratory regions. J Comp Neurol., 290: 278-288.

Bagger-Sjoback, D., Wersall, J. (1973). The sensory hairs and tectorial membrane of the basilar papilla in the lizard Calotes vesicolor. J Neurocytol., 2: 329-350.

Bang, P.I, Sewell, F.W., Malicki, J.J. (2001). Morphology and cell type hererogeneities of the inner ear epithelia in adult and juvenile zebrafish (Danio rerio). J Comp Neurol., 438: 173-190.

Becerra, M., Anadon, R. (1993). Development of the inner ear of the brown trout (Salmo trutta fario): II. Cytodifferentiation and innervation of sensory cells. J Morphol., 216: 241-257.

Becerra, M., Anadon, R., Rodrigues-Moldes, I., De Miguel, E., Caruncho, H., Corujo, A., Rodicio, C. (1990). Desarrollo del laberintoen la larva del rodaballo (Scophthalmus maximus). Actas III Congreso Nacional Acuiculture, pp. 289-294.

Campana, S.E., Neilson, J.D. (1985). Microstructure of fish otoliths. Can J Fish Aquat Sci., 42: 1014-1032.

Corwin, J.T. (1981). Postembryonic production and aging of inner ear hair cells in sharks. J Comp Neurol., 210: 541-553.

Corwin, J.T. (1983). Postembryonic growth of the macula neglecta auditory detector in the ray, Raja clavata: Continual increases in hair cell number, neural convergence and physiological sensitivity. J Comp Neurol., 217: 345356.

Edds-Walton, P.L, Fay, R.R., Highstein, S. (1999). Denderitic arbors and central projections of physiologically characterized auditory fibers from the saccule of the toadfish, Opsanus tau. J Comp Neurol., 411: 212-238.

Ernstson, S., Smith, C.A. (1986). Stereo-kinociliar bonds in mammalian vestibular organs. Acta Otolaryngol., 101: 395-402.

Flock, A., Flock, B., Murray, E. (1977). Studies on the sensory hairs of receptor cells in the inner ear. Acta Otolaryngol., 83: 85-91.
Hackney, C.M., Furness, D.N. (1995). Mechanotransduction in vertebrate hair cells: structure and function of the stereociliary bundle. Am J Physiol., 268: 113.

Haddon, C., Lewis, J. (1996). Early ear development in the embryo of the zebrafish, Danio rerio. J Comp Neurol., 365: 113-128.

Hawkins, A.D. (1986). Underwater sound and fish behavior. In: The Behavior of Teleost Fishes (Pitcher, T., Ed.). The Johns Hopkins University Press. pp $114-151$.

Hertwig, I., Schneider, H. (1999). Comparative light and electron microscopic study of the auditory organs of two species of fishes (Pisces): Hyphessobrycon simulans (Ostariophysi) and Poecilia reticulata (Acanthopterygii). Eur J Morphol., 37: 17-28.

Hudspeth, A.J. (1982). Extracellular current flow and the site of transduction by vertebrate hair cells. J Neurosci., 2: 1-10.

Hudspeth, A.J. (1983). Mechano-electrical transduction by hair cells in the acousticolateralis sensory system. Ann Rev Neorosci., 6: 187-215.

Hudspeth, A.J., Jacobs, R. (1979). Stereocilia mediate transduction in vertebrate hair cells (auditory system/cilium/vestibular system). Proc Natl Acad Sci., USA, 76: 1506-1509.

Jenkins, D.B. (1977). A light microscopic study of the saccule and lagena in certain catfishes. Am J Anat., 150: 605-630.

Kawamura, G., Ishida, K. (1985). Changes in sense organ morphology and behaviour with growth in the flounder Paralichthys olivaceus. Bull. Japan. Soc. Sci. Fish., 51: 155-165.

Kawamura, G.; Mori, H. and kuwahara, A. (1989). Comparison of sense organ development in wild and reared flounder Paralichthyes olivaceus larvae. Nippon Suisan Gakkaishi, 55: 2079-2083.

Ladich, F. and Popper, A.N. (2001). Comparison of the inner ear ultrastructure between teleost fishes using different channels for communication. Hear. Res., 154: 6272.

Lanford, P. J.; Platt, C. and Popper, A. N. (2000). Structure and function in the saccule of the goldfish (Carassius auratus): a model for diversity in the nonamniote ear. Hear Res, 143: 1-13.

Little, K. F., Neugebauer, D.-Ch. (1985). Interconnections between the stereovilli of the fish inner ear. II. Systemic investigation of saccular hair bundles from Rutilus rutilus (Teleostei). Cell Tissue Res., 242: 427-432.

Lovell, J.M., Findlay, M.M., Moate, R.M., Nedwell, J.R., Pegg, M.A. (2005). The inner ear morphology and hearing abilities of the paddlefish (Polyodon spathula) and the lake sturgeon (Acipenser fulvescens). Comp Biochem Physiol A: Mol Integr Physiol., 142, 286-296.

Lovell, J.M, Findlay, M.M., Harper, J.M., Moate, R.M. (2006). The inner ear ultrastructure from the paddlefish (Polyodon spathula) using transmission electron microscopy. J Microscopy, 222: 36-41.

Nagel, G., Neugebauer, D.-C., Schmidt, B., Thurm, U. (1991). Structures transmitting stimulatory force to the 
sensory hairs of vestibular ampullae of fishes and frog. Cell Tissue Res., 265: 567-578.

Neugebauer, D.-Ch. (1986). Interconnections between the stereovilli of the fish inner ear. III. Indications for developmental changes. Cell Tissue Res., 246: 447-453.

Neugebauer, D-C., Thurm, U. (1984). Intra-and extracellular membrane connections in stereovilli from fish inner ear. An electron microscopic study. Verh Dtsch Zool Ges., 77: 300-313.

Neugebauer, D.-Ch. and Thurm, U. (1987). Surface charges of the membrane and cell adhesion substances determine the structural integrity of hair bundles from the inner ear of fish. Cell Tissue Res., 249: 199-207.

Osborne, M.P., Comis, S.D., Pickles, J.O. (1984). Morphology and cross-linkage of streocilia in the guineapig labyrinth examined without the use of osmium as a fixative. Cell Tissue Res., 237: 43-48.

Pickles, J.O., Brix, J., Comis, C.D., Koppl, C., Osborne, M.P. (1989). The organization of the tip links and stereocilia on hair cells of bird and lizard basilar papilla. Hear Res., 41: 31-42.

Pickles, J.O., Comis, S.D., Osborne, M.P. (1984). Cross-links between stereocilia in the guinea pig organ of Corti, and their possible relation to sensory transduction. Hear. Res., 15: 103-112.

Platt, C. (1977). Hair cell distribution and orientation in goldfish otolith organs. J. Comp. Neurol., 172: 283-298.

Platt, C.T. (1983). The peripheral vestibular system in fishes. In Fish Neurobiology and Behavior. (Northcutt RG \& Davis RE, Ed.). Ann Arbor: University of Michigan Press. pp. 89-100.

Platt, C.T., Popper, A.N. (1981). Fine structure and function of the ear. In: Hearing and Sound Communication in Fishes (Tavolga, W.N., Popper, A.N., Fay, R.R., Eds.). New York: Springer-Verlag. pp. 3-38.

Platt, C. and Popper, A.N. (1984). Variation in lengths of ciliary bundles on hair cells along the macula of the sacculus in two species of teleost fishes. Scan Electr Microsc., 4: 1915-1924.

Popper, A.N. (1977). A scanning electron microscopic study of the sacculus and lagena in the ears of fifteen species of teleost fishes. J Morphol., 153: 397-418.

Popper, A.N. (1981). Comparative scanning electron microscopic investigations of the sensory epithelia in the teleost sacculus and lagena. J Comp Neurol., 200: 357374.

Popper, A.N. (2002). Structure-function relationships in the ears of fishes. Bioacoustics, 12: 115118.

Radtke, R.L., Dean, J.M. (1982). Increment formation in the otoliths of embryos, Larvae, and juveniles of the mummichog, Fundulus heteroclitus. Fish Bull., 80: 201-215.

Ramcharitar, J., Higgs, D.M., Popper, A.N. (2001). Sciaenid inner ears: A study in diversity. $\mathrm{Br}$ Beh Evolu , 58: 152-162.

Rhys Evans, P.H., Comis, S.D., Osborne, M.P., Pickles, J.O., Jefferies, D.R. (1985). Cross-links between stereocilia in the human organ of Corti. J Laryngol., 99: 1120.

Ross, M. (1974). The tectorial membrane of the rat. Am J Anat., 139: 449-482.

Saidel, W.M., Popper A.N. (1987). Sound perception in two anabantoid fishes. Comp Biochem Physiol., 88 : 37 -44 .

Saitoh, S., Yamada, J. (1989). Ultrastructure of the saccular epithelium and the otolithic membrane in relation to otolith growth in tilapia, Oreochromis niloticus (Teleostei, Cichlidae). Trans Microsc Soc., 108: 223-238.

Salem, M.A. (2003). Electron microscopic study on the sensory hair bundles and the interconnections between the stereocilia in the saccular macula of the Oreochromis aureus inner ear. J Egypt Ger Soc Zool., 42 (B): 85-106.

Salem, M.A., Al-Jahdali, M.O. (2006). Development, structure and function of the sense organs in the brownmarbled grouper Epinephelus fuscoguttatus (Teleostei: Serranidae). J Egypt Soci Biotech Environ Sci , 8: 95-138.

Salem, M.A., Omura, Y. (1998). Light and electron microscopic studies on the development of the inner ear and otolith of the ayu Plecoglossus altivelis. Fisheries Science, 64: 259-264.

Salem, M.A., Zaghloul, M.S. (2001). Structure and development of the saccular sensory epithelium in relation to otolith growth in the perch Perca fluviatilis (Teleostei). Egyptian J Biol., 3: 1-12.

Satir, P. (1977). Microvilli and cilia: Surface specializations of mammalian cells. In: Mammalian cell membranes (Jamieson, G.A., Roninson, D.N, Eds.), Vol. II, Butterworths, London, Boston, pp. 323-353.

Saunders, J.C. and Dear, S.P. (1983). Comparative morphology of stereocilia. In: Hearing and Other Senses (Fay, R.R., Gourevitch, G. Eds.). Groton: Amphora Press, Conn., pp. 175-198.

Schmidt, B., Thurm, U. (1984). Structure transmitting stimulatory force to the sensory hairs (stereovilli) of the frog sacculus. Verth Dtsch Zool. Ges., 77: 316-326.

Sokolowski, B.H.A. (1986). Development of the otolith in embryonic fishes with special references to the toadfish, Opsanus tau. Scan Elect Microsc., 4: 1635-1648.

Sokolowski, B.H.A., Popper, A.N. (1987). Gross and ultrastructural development of the saccule of the toadfish, Opsanus tau. J Morphol, 194: 323-348.

Song, J., Mathieu, A., Soper, R.F., Popper, A.N. (2006). Structure of the inner ear of nbluefin tuna Thunnus thymus. J fish Biology, 68: 1767-1781.

Thomot, A., Bauchot, R. (1987). L' organogenesis du labyrinthe membraneux chez Polypterus senegalus Cuvier, 1829. (Pisces, Holostei, Polypteridae). Anat Anz., 164: 189-211.

Waterman, E.R., Bell, D.H. (1984). Epithelial fusion during early semicircular canal formation in the embryonic zebrafish, Brachydanio rerio. Anat Rec., 210: 101-114. 